\title{
Baricitinib restrains the immune dysregulation in patients with severe COVID-19
}

\author{
Vincenzo Bronte, ${ }^{1}$ Stefano Ugel, ${ }^{1}$ Elisa Tinazzi, ${ }^{2}$ Antonio Vella, ${ }^{1}$ Francesco De Sanctis, ${ }^{1}$ Stefania Canè, ${ }^{1}$ Veronica Batani, ${ }^{1}$ \\ Rosalinda Trovato, ${ }^{1}$ Alessandra Fiore, ${ }^{1}$ Varvara Petrova, ${ }^{1}$ Francesca Hofer, ${ }^{1}$ Roza Maria Barouni, ${ }^{1}$ Chiara Musiu, ${ }^{1}$ Simone Caligola, \\ Laura Pinton, ${ }^{1}$ Lorena Torroni, ${ }^{3}$ Enrico Polati, ${ }^{4}$ Katia Donadello, ${ }^{4}$ Simonetta Friso, ${ }^{2}$ Francesca Pizzolo, ${ }^{2}$ Manuela lezzi, ${ }^{5}$ \\ Federica Facciotti, ${ }^{6}$ Pier Giuseppe Pelicci, ${ }^{6}$ Daniela Righetti, ${ }^{7}$ Paolo Bazzoni, ${ }^{7}$ Mariaelisa Rampudda, ${ }^{7}$ Andrea Comel, ${ }^{7}$ \\ Walter Mosaner, ${ }^{7}$ Claudio Lunardi, ${ }^{2}$ and Oliviero Olivieri ${ }^{2}$
}

IImmunology Section, Department of Medicine, IInternal Medicine Section B, Department of Medicine, 3Unit of Epidemiology and Medical Statistics, Department of Diagnostics and Public Health, University and Hospital Trust of Verona, Verona, Italy. ${ }^{4}$ Intensive Care Unit, Department of Surgery, Dentistry, Maternity and Infant, University and Hospital Trust of Verona, Verona, Italy. ${ }^{5}$ Center for Advanced Studies

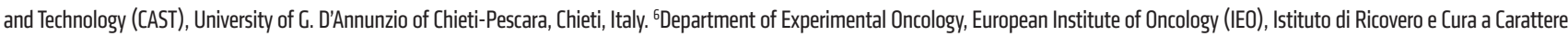
Scientifico (IRCCS), Milan, Italy. Pederzoli Hospital, Peschiera sul Garda, Italy.

BACKGROUND. Patients with coronavirus disease 2019 (COVID-19) develop pneumonia generally associated with lymphopenia and a severe inflammatory response due to uncontrolled cytokine release. These mediators are transcriptionally regulated by the JAK/ STAT signaling pathways, which can be disabled by small molecules.

METHODS. We treated a group of patients $(n=20)$ with baricitinib according to an off-label use of the drug. The study was designed as an observational, longitudinal trial and approved by the local ethics committee. The patients were treated with $4 \mathrm{mg}$ baricitinib twice daily for 2 days, followed by $4 \mathrm{mg}$ per day for the remaining 7 days. Changes in the immune phenotype and expression of phosphorylated STAT3 (p-STAT3) in blood cells were evaluated and correlated with serum-derived cytokine levels and antibodies against severe acute respiratory syndrome-coronavirus 2 (anti-SARS-CoV-2). In a single treated patient, we also evaluated the alteration of myeloid cell functional activity.

RESULTS. We provide evidence that patients treated with baricitinib had a marked reduction in serum levels of IL-6, IL-1 3 , and TNF- $\alpha$, a rapid recovery of circulating $T$ and $B$ cell frequencies, and increased antibody production against the SARS-CoV- 2 spike protein, all of which were clinically associated with a reduction in the need for oxygen therapy and a progressive increase in the $\mathrm{P} / \mathrm{F}\left(\mathrm{PaO}_{2}\right.$, oxygen partial pressure/ $\mathrm{FiO}_{2}$, fraction of inspired oxygen) ratio.

CONCLUSION. These data suggest that baricitinib prevented the progression to a severe, extreme form of the viral disease by modulating the patients' immune landscape and that these changes were associated with a safer, more favorable clinical outcome for patients with COVID-19 pneumonia.

TRIAL REGISTRATION. ClinicalTrials.gov NCT04438629.

FUNDING. This work was supported by the Fondazione Cariverona (ENACT Project) and the Fondazione TIM.

\section{Introduction}

The pandemic spread of a novel, highly pathogenic coronavirus, severe acute respiratory syndrome-coronavirus 2 (SARS-CoV-2), has found the international medical community largely unprepared in terms of prophylactic and therapeutic measures (1). The resulting syndrome, known as coronavirus disease 2019 (COVID-19), is characterized by

\section{Related Commentary: p. 6225}

Authorship note: VB and SU contributed equally to this work. Conflict of interest: The authors have declared that no conflict of interest exists. Copyright: (5) 2020, American Society for Clinical Investigation. Submitted: June 26, 2020; Accepted: August 17, 2020; Published: November 3, 2020. Reference information: J Clin Invest. 2020;130(12):6409-6416. https://doi.org/10.1172/JCl141772. a profound dysfunction of the upper and lower respiratory tract, with severity ranging from mild to moderate respiratory failure, to acute respiratory distress syndrome (ARDS) (2). Recently, the crucial role of the cytokine release syndrome (CRS) (3), also referred to as "cytokine storm," in acute lung damage and $\operatorname{ARDS}(4,5)$ has become evident, thus providing the theoretical ground for therapeutic approaches able to interfere with the inflammatory cascade. Indeed, although the majority of patients who are either asymptomatic or in early stages of the disease are able to clear the infection, some with moderate disease requiring hospitalization progress to a clinically severe phase associated with the cytokine storm within 10 days from symptom onset. These observations suggest that in some patients the immune response might be skewed and unable to neutralize the effects of the viral infection. For this reason, in addition to antiviral therapy, immune modulators of cytokine production have been advanced. 


\section{Table 1. Clinical characteristics of the patients during treatment}

\section{Characteristic}

\begin{tabular}{|c|c|c|c|c|c|c|c|c|c|c|c|}
\hline $\begin{array}{l}\text { Characteristic } \\
\text { Clinical, laboratory, } \\
\text { respiratory parameters } \\
\text { during treatment }\end{array}$ & \multicolumn{5}{|c|}{ Barıcıtınıb } & \multicolumn{5}{|c|}{ No baricitinib } & $\begin{array}{c}P \text { value } \\
P \text { value } \\
\text { to Baricitinib } \\
\text { vs. to No baricitinib }\end{array}$ \\
\hline $\begin{array}{l}\text { Respiratory rate ( } n / \mathrm{min}) \text {, } \\
\text { median (IQR) }\end{array}$ & $\begin{array}{c}19 \\
(16.25-24)\end{array}$ & $\begin{array}{c}17.5 \\
(15-19)\end{array}$ & $\begin{array}{c}16 \\
(15-18)\end{array}$ & 0.04 & 0.02 & $\begin{array}{c}22 \\
(20-30)\end{array}$ & $\begin{array}{c}22 \\
(20-28)\end{array}$ & $\begin{array}{c}20 \\
(18-30)\end{array}$ & 0.64 & 0.30 & 0.01 \\
\hline $\mathrm{P} / \mathrm{F}$, median (IQR) & $\begin{array}{c}241 \\
(200-295.8)\end{array}$ & $\begin{array}{c}290 \\
(248-319)\end{array}$ & $\begin{array}{c}331 \\
(287.5-367.3)\end{array}$ & 0.08 & 0.02 & $\begin{array}{c}220 \\
(128.5-319)\end{array}$ & $\begin{array}{c}141 \\
(86.25-215.3)\end{array}$ & $\begin{array}{c}225 \\
(150-281.0)\end{array}$ & 0.02 & 0.3 & 0.57 \\
\hline $\begin{array}{l}\mathrm{FiO}_{2} \text { required (\%), median } \\
\text { (IQR) }\end{array}$ & $\begin{array}{c}29.5 \\
(25-3425)\end{array}$ & $\begin{array}{c}28 \\
(24-40)\end{array}$ & $\begin{array}{c}22.5 \\
(21-28)\end{array}$ & 0.59 & $<0.001$ & $\begin{array}{c}31 \\
(21-50)\end{array}$ & $\begin{array}{c}36 \\
(28-60)\end{array}$ & $\begin{array}{c}28 \\
(21-70)\end{array}$ & $<0.001$ & 0.08 & 0.70 \\
\hline CRP (mg/L), median (IQR) & $\begin{array}{c}53.15 \\
(43.08-77.63)\end{array}$ & $\begin{array}{c}13.9 \\
(7.83-22.75)\end{array}$ & $\begin{array}{c}9.7 \\
(4.33-14.23)\end{array}$ & $<0.001$ & $<0.001$ & $\begin{array}{c}64.5 \\
(37-130.3)\end{array}$ & $\begin{array}{c}705 \\
(43-75)\end{array}$ & $\begin{array}{c}38 \\
(12-6325)\end{array}$ & 0.87 & 0.12 & 0.16 \\
\hline Fever $\left({ }^{\circ} \mathrm{C}\right)$, median (IQR) & $\begin{array}{c}36.9 \\
(36.25-38)\end{array}$ & $\begin{array}{c}36.2 \\
(36.2-36.7)\end{array}$ & $\begin{array}{c}36.4 \\
(36.05-36.6)\end{array}$ & 0.003 & 0.010 & $\begin{array}{c}37.2 \\
(36.48-38)\end{array}$ & $\begin{array}{c}36 \\
(36-36.33)\end{array}$ & $\begin{array}{c}36 \\
(36-36.48)\end{array}$ & 0.006 & 0.007 & 0.57 \\
\hline
\end{tabular}

$\mathrm{P} / \mathrm{F}, \mathrm{PaO}_{2}$, oxygen partial pressure/ $\mathrm{FiO}_{2}$, fraction of inspired oxygen.

Baricitinib is an oral, selective, and reversible inhibitor of the Janus kinases JAK1 and JAK2 that was previously shown to dampen inflammatory immune responses and approved for indications such as rheumatoid arthritis (RA) (6). The drug was licensed for administration at a daily oral dose of $2 \mathrm{mg}$ with good results in terms of clinical response and safety (7). In a recent meta-analysis, no statistically significant increase in the risk of serious infections was recognized over a long treatment period (8), thus the use of this agent for a short 14-day period should have "trivial" adverse activity (9). In addition to the potential cytokine-inhibitory activity, baricitinib was predicted to inhibit angiotensin-converting enzyme-mediated (ACE-mediated) endocytosis of SARS-CoV-2 by machine-learning algorithms (9).

We hypothesized that JAK/STAT pathway inhibition might prevent the progression toward a severe/extreme form of the viral disease by modulating the patients' immune response. Here we provide evidence that baricitinib-induced changes in the immune landscape were associated with a favorable clinical outcome for patients with COVID-19-related pneumonia.

\section{Results}

Baricitinib improves the clinical parameters of SARS-CoV-2 infection. To understand the clinical impact of baricitinib on COVID-19, we assessed 20 patients who were admitted from March 18 to April 18, 2020, to the Unit of Internal Medicine at the University Hospital of Verona and Pederzoli Hospital of
Peschiera with a diagnosis of COVID-19 pneumonia, which was confirmed by a nasal swab positive for SARS-CoV-2 by reverse transcriptase PCR (RT-PCR) assay.

In total, 88 patients (44 males, 44 females) affected by COVID-19-related pneumonia were hospitalized during the study period. All participants were treated with either hydroxychloroquine or antiviral therapy (lopinavir/ritonavir) as single agents or in combination (hydroxychloroquine plus antiviral therapy) depending on the clinical features. Supportive therapy, such as antibiotic prophylaxis and anticoagulant treatment, was provided at the discretion of the clinicians (Supplemental Table 1; supplemental material available online with this article; https://doi. org/10.1172/JCI141772DS1). Steroid therapy was systematically avoided. However, 12 (6 males, 6 females) of these patients were excluded from the analysis because of their positive, active history of malignancies: 2 patients had hematological disorders (1 with multiple myeloma and 1 with acute myeloid leukemia), and 10 patients had solid malignancies, including lung and breast cancers as well as kidney, prostate, ovarian, and gastrointestinal tumors. Arterial hypertension and cardiovascular disease as well as diabetes, chronic obstructive pulmonary disease, and chronic kidney disease were the prevalent morbidities for the other 76 patients who were ultimately enrolled in the study (Supplemental Table 1). Among them, 20 patients received the full course of baricitinib according to the study protocol. The other 56 patients were considered the control group, since they never received baricitinib,

\section{Table 2. Primary outcomes of treated patients}

$\begin{array}{lccc}\text { Primary outcomes } & \text { Baricitinib } & \text { No baricitinib } & P \text { value } \\ \text { Deaths, } n(\%) & 1(5) & 25(45) & <0.001 \\ \text { Incidence of ARDS, } n(\%) & 3(15) & 15(27) & 0.37 \\ \text { Duration of hospitalization, days (median: min max) } & 12(5-24) n=19 & 11(3-46) n=31 & 0.28\end{array}$

min, minimum; max, maximum. 
neither as a full course nor as partial treatment. According to the inclusion criteria and baricitinib pharmacokinetics, patients were treated with $4 \mathrm{mg}$ baricitinib twice daily for 2 days followed by $4 \mathrm{mg}$ per day for the remaining 7 days. A low dose of $2 \mathrm{mg}$ twice daily for 2 days followed by $2 \mathrm{mg}$ per day was maintained for patients older than 75 years. A dose reduction was also considered in instances of renal insufficiency (glomerular filtration rate [GFR] $<30 \mathrm{~mL} /$ $\min / 1.73 \mathrm{~m}^{2}$ ), hepatotoxicity, or myelotoxicity. Of note, during the hospitalization, the patients enrolled in the baricitinib group did not experience any type of bacterial or mycotic infections, and, overall, none of the patients developed deep vein thrombosis or pulmonary thromboembolism.

Patients included in the baricitinib-treated group were similar to those included in the control group for age, sex, and comorbidities and for several clinical feature values (Supplemental Table 1). Indeed, between the 2 patient cohorts, we observed no differences in the symptoms ascribed to COVID-19, such as fever and cough. Moreover, patients in the 2 groups were clinically similar with regard to several respiratory parameters, such as respiratory frequency, $\mathrm{P} / \mathrm{F}\left(\mathrm{PaO}_{2}\right.$, oxygen partial pressure/ $\mathrm{FiO}_{2}$, fraction of inspired oxygen) ratio, and need for oxygen replacement therapy (Table 1), although patients in the baricitinib treatment group had a radiologic score reflecting more severe disease. The laboratory parameters were homogeneous between the 2 groups except for lactate dehydrogenase (higher in the baricitinib-treated group) and D-dimer (lower in the baricitinib-treated group) levels (Table 1 ).

Clinically, the outcome for the cohort of baricitinib-treated patients was different in terms of mortality. Among the baricitinib-treated patients, 1 of 20 (5\%) died after completion of the therapeutic treatment regimen compared with 25 (45\%) of 56 patients in the non-baricitinib-treated group $(P<0.001)$ (Table 2$)$. We observed no significant difference in ARDS incidence or disease duration, expressed as the number of hospitalization days (Table 2). Finally, for an in-depth evaluation of the impact of baricitinib treatment on the resolution of COVID-19 pathology, we analyzed the clinical features of both baricitinib-treated patients and control patients at the time of enrollment $(t 0)$ and $4(t 4)$ and $7(t 7)$ days after treatment (Table 1). Interestingly, patients treated with baricitinib experienced a faster reduction in the need for oxygen flow therapy $(P<0.001)$ and a more rapid increase in the $\mathrm{P} / \mathrm{F}$ ratio compared with the control group $(P=0.02)$, as well as a reduction in serum levels of $C$-reactive protein $(\mathrm{CRP})(P<0.001)$, whereas no differences in fever resolution were observed between the 2 groups (Table 1). When we considered interstitial lung involvement, either chest x-ray or high-resolution CT (HRCT) revealed variable extension at different disease stages. We observed an increase in interstitial involvement in the control group on the fourth day of treatment and a reduction of the same on the seventh day of treatment in both groups, with no statistically relevant differences. Since at the time of hospital admission the interstitial lung involvement was more frequent and extensive in patients enrolled in the baricitinib treatment group compared with patients in the control cohort, we speculated about the possible clinical benefit to the lungs conferred by baricitinib treatment (data not shown). Collectively, these data indicate a clinical benefit of baricitinib treatment for patients with COVID-19 and support further randomized, controlled trials.
Baricitinib affects the immune landscape in patients with COVID-19. In order to evaluate the downstream molecular targets of baricitinib activity, we first demonstrated that patients with COVID-19-related pneumonia expressed significantly higher levels of phosphorylated STAT3 (p-STAT3) (Tyr705) in different leukocyte subsets compared with healthy donors (HDs) (Supplemental Figure 1 and Supplemental Figure 2A). However, we did not detect a significant increase in p-STAT1 (Tyr701) expression in circulating leukocytes isolated from patients with COVID-19 compared with expression in HDs (Supplemental Figure 2A). While the viral load should have triggered a type I IFN response, which relies on STAT1 signaling, the use of an antibody restricted to p-Tyr701 and that does not detect p-Ser727 could have limited the ability to follow STAT1 changes in the samples. Therefore, p-STAT3 was selected to monitor the on-target effect of the drug in vivo. Indeed, baricitinib administration induced significant inhibition of p-STAT3 in T lymphocytes (Supplemental Figure 2B), NK cells (Supplemental Figure 2C), monocytes (Supplemental Figure 2D), and neutrophils (Supplemental Figure 2E), as observed in 6 patients. We detected a significant reduction in p-STAT3 already 4 days after administration of the drug, indicating that the treatment achieved effective on-target activity. Conversely, we observed no statistically relevant activity in $\mathrm{B}$ cells during the treatment period (Supplemental Figure 2F).

We then analyzed different immune cell populations in the blood of patients who received baricitinib versus those who did not. In 12 baricitinib-treated patients, we detected no modification in the absolute number of circulating leukocytes (Supplemental Figure 3A) as compared with control group $(n=8)$. These patients presented the same clinical features at baseline except for the median $\mathrm{P} / \mathrm{F}(P=$ $0.04)$ and LDH $(P<0.01)$, which were respectively lower and higher in the baricitinib-treated patients (data not shown). Remarkably, all baricitinib-treated patients showed an incremental increase in the absolute number of circulating lymphocytes during the treatment period, reaching the reference range $(1200-2000$ cells $/ \mu \mathrm{L})$ by the end of the treatment $(t 7)$ (Figure 1A). Interestingly, baricitinib treatment increased the number of circulating $\mathrm{T}$ cells (Figure 1B) and $\mathrm{B}$ cells (Figure 1C). We especially observed a significant effect of the drug on circulating CD4 ${ }^{+} \mathrm{T}$ cells (Figure 1D) and, among them, lymphocytes with an effector memory phenotype $\left(\mathrm{CD}^{+} \mathrm{CD} 4^{+} \mathrm{C}\right.$ $\mathrm{D} 45 \mathrm{RA}^{-} \mathrm{CD}^{-} 7^{-}$) were particularly expanded (Figure 1E and Supplemental Figure 4). On the other hand, we found that the absolute number of $\mathrm{CD}^{+} \mathrm{T}$ lymphocytes was lower than the reference range in both baricitinib-treated and control groups (Figure $1 \mathrm{~F}$ ). Baricitinib did not affect the absolute number of NK cells (Supplemental Figure 3B) or neutrophils (Supplemental Figure 3C). Notably, in both untreated and baricitinib-treated patients, we observed an expansion of monocytes by day 7, which probably reflects a common COVID-19 evolution (Supplemental Figure 3D), as recently published (10). Using t-distributed stochastic neighbor embedding (t-SNE) analysis, we confirmed the incremental increase in naive (from $11.2 \%$ to $13.8 \%$ ) and central memory (from $11.9 \%$ to $16.7 \%$ ) $\mathrm{CD}^{+} \mathrm{T}$ cell populations and B lymphocytes (from $11.8 \%$ to $15.7 \%$ ), but also unveiled a shift among the $\mathrm{CD} 8^{+} \mathrm{T}$ cells after baricitinib treatment. Specifically, there was a time-dependent decrease in senescent cells $\left(\mathrm{CD} 8{ }^{+} \mathrm{CD} 45 \mathrm{RA}^{+} \mathrm{CD} 57^{+} \mathrm{CD} 27\right.$; from $7.3 \%$ to $\left.3.3 \%\right)$, with a concomitant increase in both naive $\left(\mathrm{CD}^{+} \mathrm{CD} 45 \mathrm{RA}^{+} \mathrm{CD} 57^{-} \mathrm{CD} 27^{+}\right.$; from $4.3 \%$ to $5.3 \%$ ) and memory $\left(\mathrm{CD}^{+} \mathrm{CD}^{+} \mathrm{CD} 27^{+} \mathrm{CD} 45 \mathrm{RA}-\right.$, from $3.4 \%$ 
A

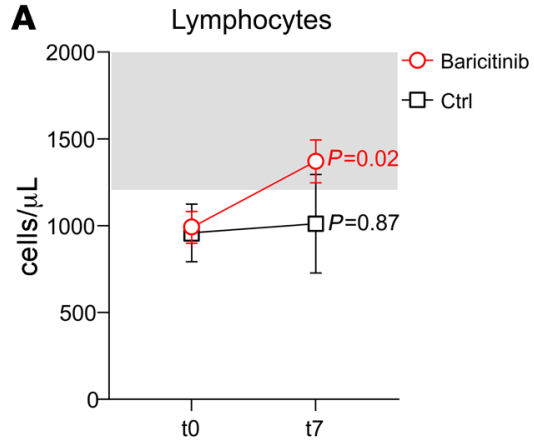

D $\quad \mathrm{CD}^{+} \mathrm{T}$ Lymphocytes

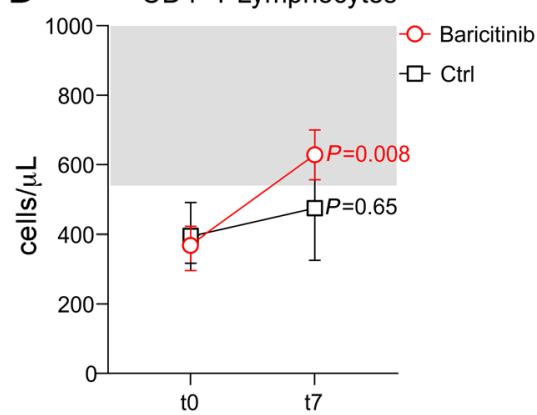

G

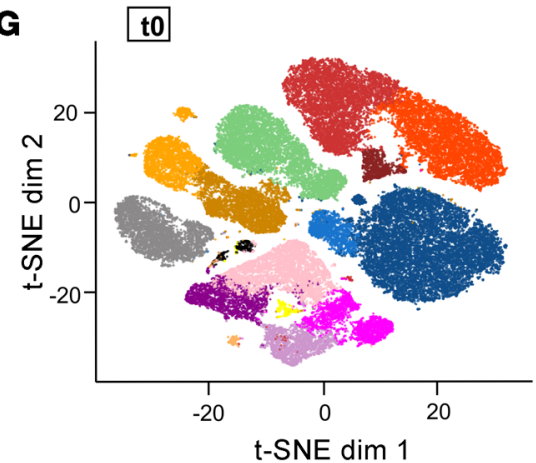

B

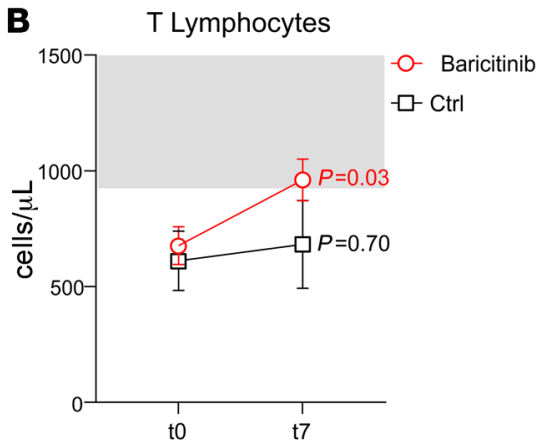

E
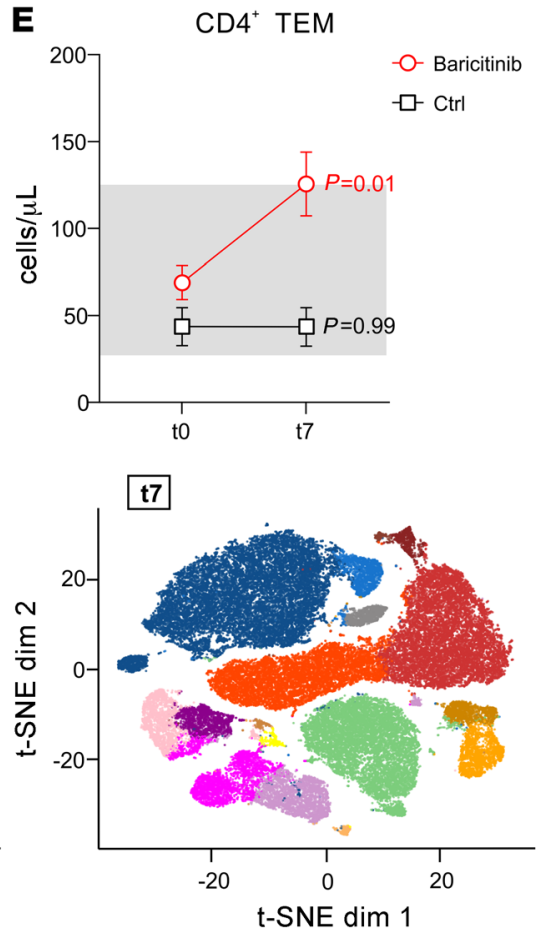

C B Lymphocytes

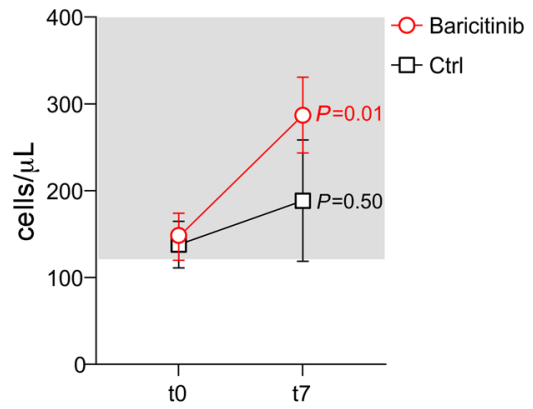

F $\quad \mathrm{CD} 8^{+} \mathrm{T}$ Lymphocytes

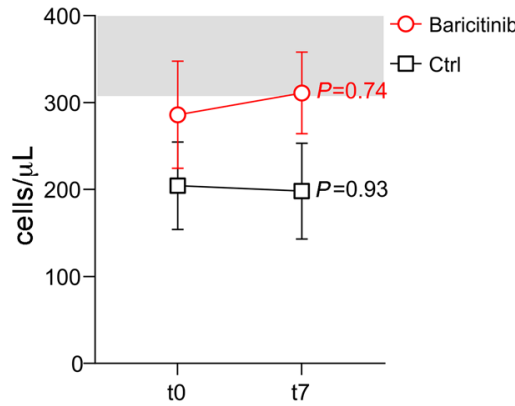

- Monocytes (t0 $20.6 \%$, t7 25.9\%)

O Monocytes (CD16 ; t0 2.9\%, t7 2.7\%)

O Residual PMNs (CD16 $6^{\text {high }}$; t0 $7.5 \%$, t7 1.7\%)

O lymphocytes (to $11.8 \%$, t7 15.7\%)

- $\mathrm{CD}^{+}$TCM (t0 $11.9 \%, \mathrm{t} 7$ 16.7\%)

- $\mathrm{CD}^{+}$TEM (t0 $2.0 \%, \mathrm{t} 71.0 \%$ )

- $\mathrm{CD}^{+} \mathrm{T}$ naive (t0 $11.2 \%, \mathrm{t} 713.8 \%$ )

O CD4 $4^{+}$TEMRA (to N/A, t7 0.3\%)

O CD8 ${ }^{+}$TM (t0 $3.4 \%$, t7 $4.8 \%$ )

- CD8 $8^{+}$TEM (t0 $3.6 \%$, t7 $2.5 \%$ )

- CD8 ${ }^{+}$T naive (t0 $4.3 \%$, t7 $5.3 \%$ )

O CD8 ${ }^{+}$T senescent (t0 $7.3 \%$, t7 3.3\%)

O NKT (to $0.3 \%$, t7 $0.3 \%$ )

O NKT (to $0.6 \%$, t7 $0.5 \%$ )

- NKT senescent (t0 $0.7 \%$, t7 N/A)

NK senescent (t0 $4.8 \%$, t7 3.1\%)

O NK (to $7.0 \%$, t7 $2.1 \%$ )

Figure 1. Baricitinib treatment restores normal lymphocyte counts in the blood. Peripheral blood from patients with COVID-19 enrolled in either the baricitinib $(n=12)$ or basic treatment $(n=8, C$ trl) arm was analyzed by flow cytometry at $t 0$ (baseline) and $t 7$ ( 7 days after treatment). The number of cells per microliter is reported for lymphocytes (A), T lymphocytes (B), B lymphocytes (C), CD4+ T lymphocytes (D), CD4+ T effector memory (TEM) cells (E), and $\mathrm{CD}^{+} \mathrm{T}$ lymphocytes $(\mathbf{F})$. The normal reference range is shown in the light gray boxes. Data are reported as the mean $\pm \mathrm{SEM}$. Statistical analysis was determined by 1-way, repeated-measures ANOVA. (G) t-SNE analysis of peripheral blood from 12 baricitinib-treated patients at $t 0$ (left) and $t 7$ (right). The following identified clusters are shown in different colors: monocytes, CD16 ${ }^{+}$monocytes, residual polymorphonuclear neutrophils (PMNs) (CD16 ${ }^{\text {hi }}$, $B$ lymphocytes (CD19+CD45RA ${ }^{+}$), CD4+ $T$ central memory (TCM) cells (CD3+CD4+CD27+CD45RA $)$, CD4+ TEM cells (CD3+CD4+CD57+CD27-CD45RA-), CD4 ${ }^{+}$naive T cells

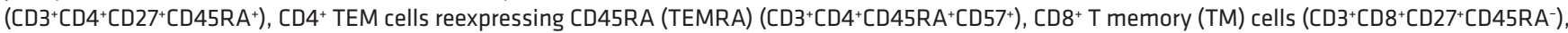

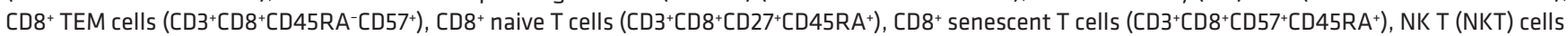

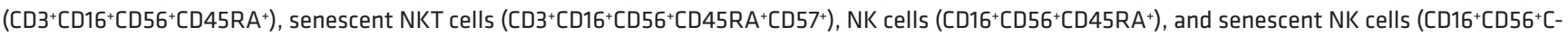
D45RA ${ }^{+}\left(D 57^{+}\right)$. Ctrl, control; dim, dimensionality.

to $4.8 \%)^{-C D^{+}} \mathrm{T}$ lymphocytes, suggesting an effect of baricitinib in supporting effector $\mathrm{T}$ cell activation (Figure $1 \mathrm{G}$ ). To validate our t-SNE analysis, each marker was extracted using functions in flowCore (Supplemental Figure 5). Conversely, we did not detect chang-

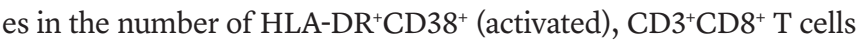
(11). It remains to be determined whether the $\mathrm{CD} 8^{+} \mathrm{T}$ cell function and/or repertoire might be altered by the treatment.

Considering the variation in B lymphocyte numbers (Figure 1C), we also evaluated the plasma levels of IgA and IgG specific for the receptor-binding domain (RBD) of the SARS-CoV-2 spike protein. As shown in Figure 2, A and B, although we did not observe a baricitinib-specific effect on IgA levels between $t 0$ and $t 7$, a significant increase in IgG was present only in the baricitinib-treated group. Among the patients who did not present any virus-specific IgG at $t 0$ in the baricitinib-treated group $(n=20), 8$ of 9 of these individuals developed high titers at $t 7$. The patient who was never found to have virus-specific IgG was the only death in the baricitinib treatment group. In contrast, in the control group $(n=8)$, among the 7 patients who did not show any virus-associated IgG at $t 0$, only 3 developed virus-specific IgG.

We next demonstrated that baricitinib treatment reduced the plasma concentration of several proinflammatory cytokines, 

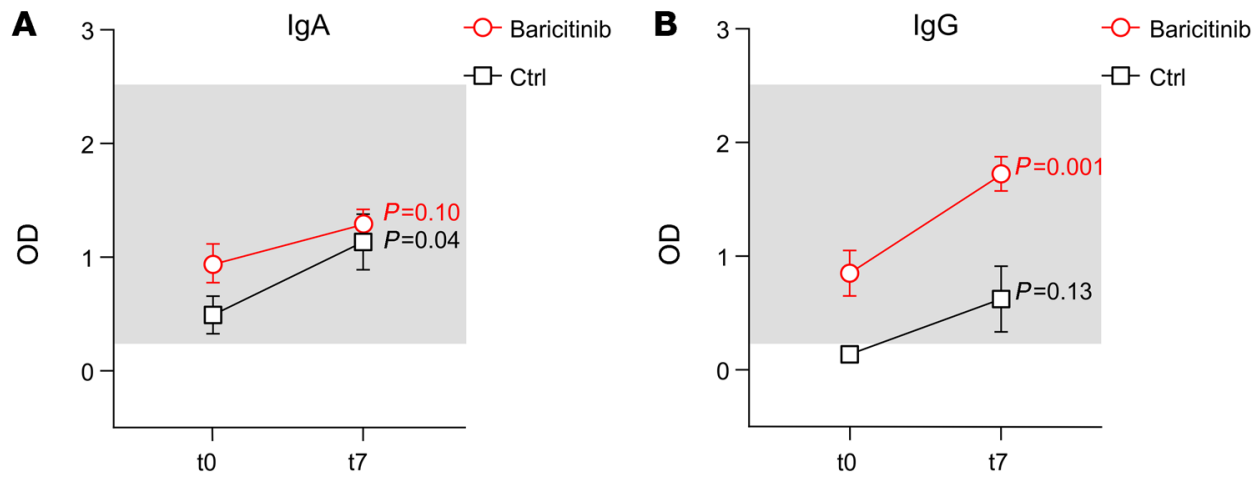

Figure 2. Baricitinib treatment affects IgC levels and the production of inflammatory cytokines that contribute to the cytokine storm. Plasma from patients with COVID-19 enrolled in either the baricitinib ( $n=$ $20)$ or basic $(n=8)$ treatment arm was analyzed at $t 0$ (baseline) and $t 7$ (7 days after treatment) to measure the concentrations of IgA (A), IgC (B), IL-1 $\beta$ (C), IL-6 (D), TNF- $\alpha$ (E), and IL-8 (F). For serological data, the light gray boxes identify the range of $A b$ detection. The normal median value for cytokines is indicated in the light gray boxes. Data are presented as the mean \pm SEM.
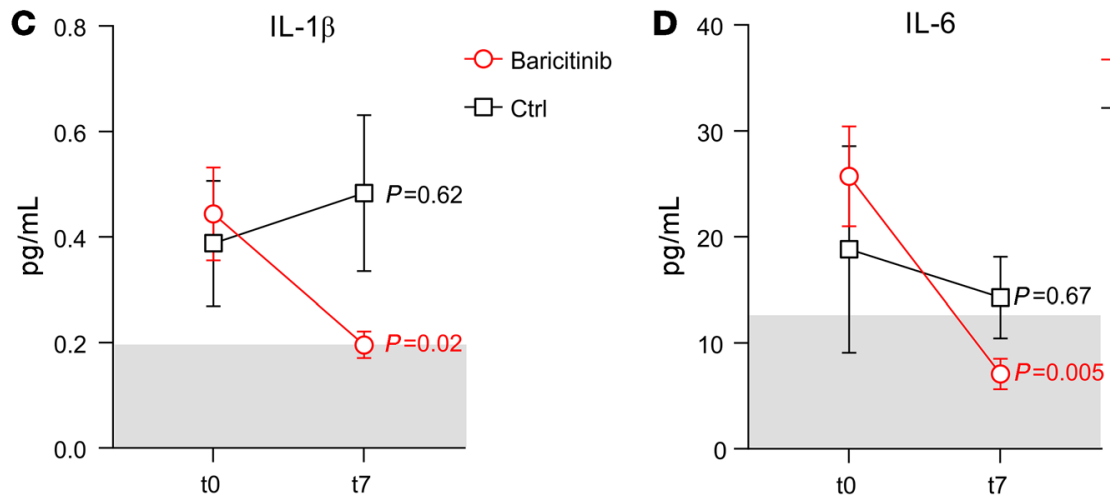
- Baricitinib Statistical analysis was determined by 1-way, repeated-measures ANOVA.
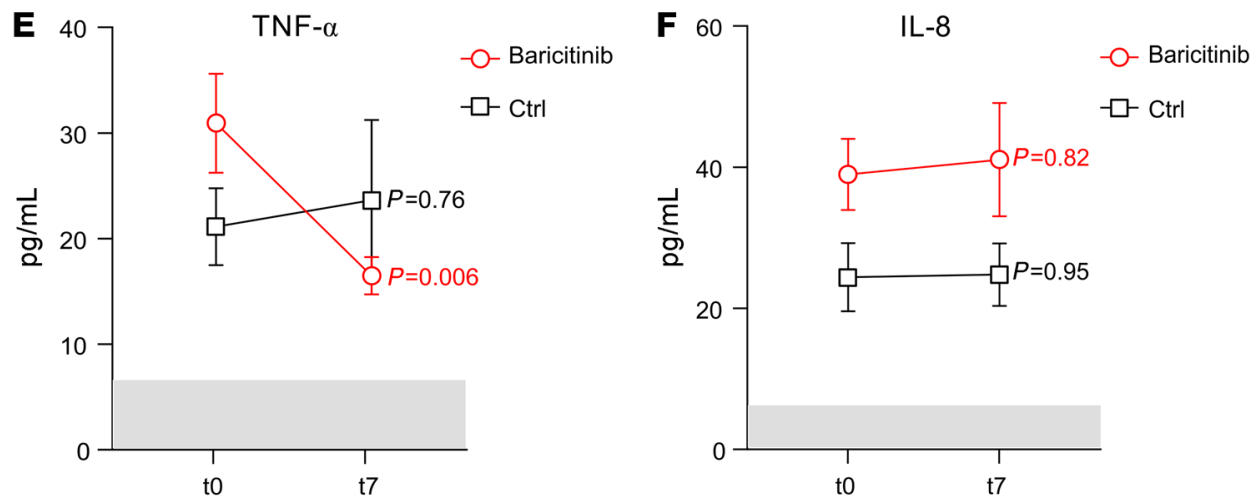

which are produced at abnormal levels in patients with COVID-19 as well as in patients with CRS. Indeed, 7 days after the first dose, we detected a significant reduction in IL-1 $\beta$, IL-6, and TNF- $\alpha$ plasma concentrations in the baricitinib-treated patients but not in the control group (Figure 2, C-E), supporting the idea that baricitinib is an effective therapeutic tool against cytokine storm, a major cause of ARDS and multiorgan failure in patients with COVID-19 (4). Interestingly, there were no differences in IL-8 concentrations, indicating that JAK1/JAK2-dependent molecular pathways are not the main regulators of IL-8 production, at least not in these patients (Figure 2F).

Baricitinib modifies immune-suppressive features of myeloid cells. Although the efficacy of baricitinib treatment was assessed as a decrease in the intensity of p-STAT3 and levels of proinflammatory cytokines, we hypothesized that these alterations might also impact the ability of myeloid cells to modulate $\mathrm{T}$ cell proliferation. To verify this, we studied in detail the case of a 68-year-old woman who was admitted to the hospital for the persistence of fever and dyspnea. Lung $\mathrm{x}$-ray analysis revealed bilateral and interstitial pneumonia, compatible with positive SARS-CoV-2, which was further confirmed by an oropharyngeal swab. The patient presented with a rapidly worsening clinical course that required admission to the intensive care unit (ICU). Upon hospitalization, she agreed to receive standard antiviral treatment and off-label baricitinib, both of which were continued in the ICU. After 1 week in the ICU, the patient began to breath spontaneously and was transferred to the pneumology unit. Ten days later, she was released from the hospital in good clinical condition. From the peripheral blood of the patient, we isolated monocytes (CD14+ cells), low-density neutrophils (LDNs), or normal-density neutrophils (NDNs) at 2 different time points of hospitalization: during her ICU stay (ICU) and when she left the ICU (No ICU). At the same time, we evaluated the serum levels of several proinflammatory cytokines and detected a decrease in IL- $1 \beta$ and TNF- $\alpha$ levels from the beginning of the treatment (day $0, t 0$ ) until the end (day $7, t 7)$. Interestingly, IL- 6 levels increased by $t 4$ but dropped completely by $t 7$, where- 
A
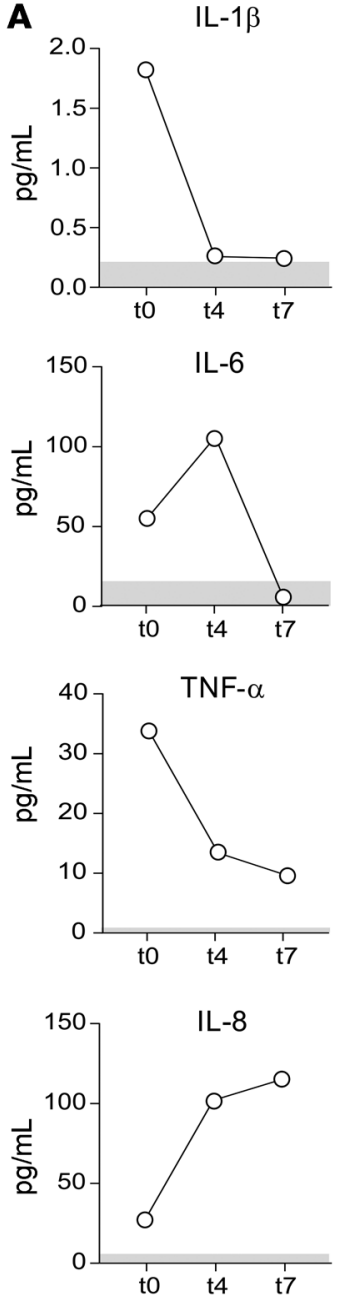

B

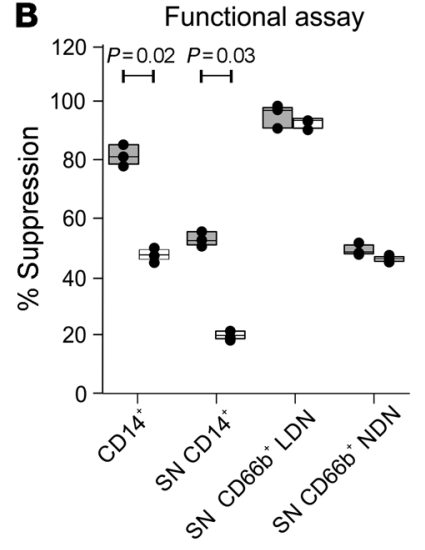

C
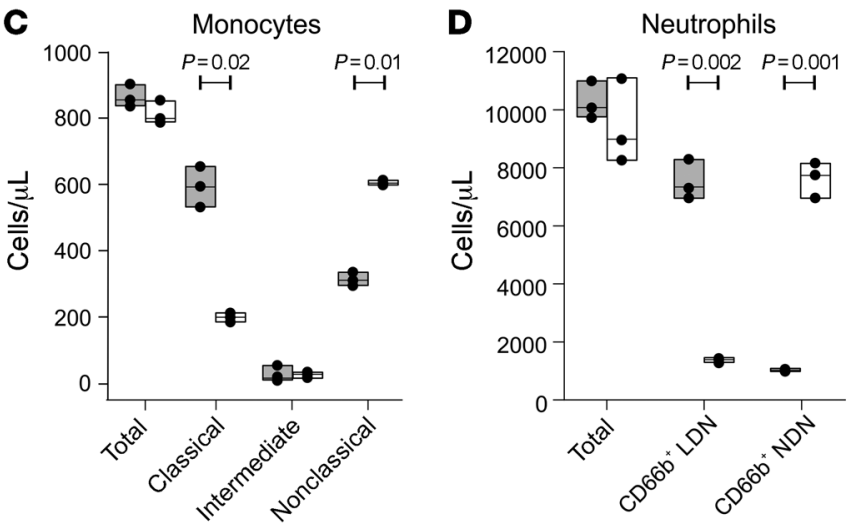

$\square$ ICU $\square$ NoICU
$\mathbf{E}$

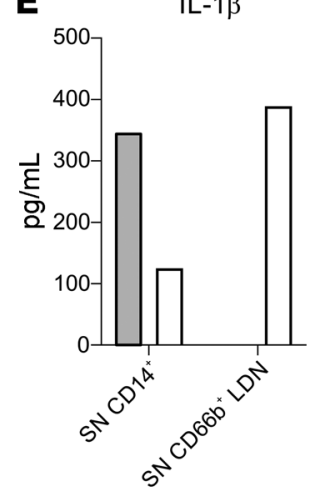

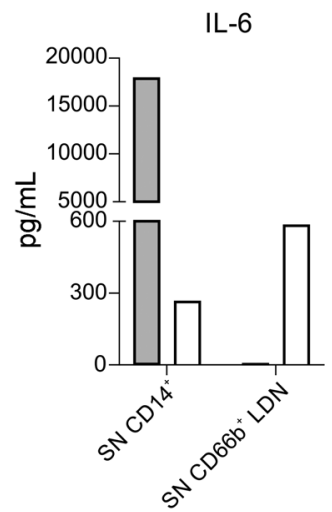
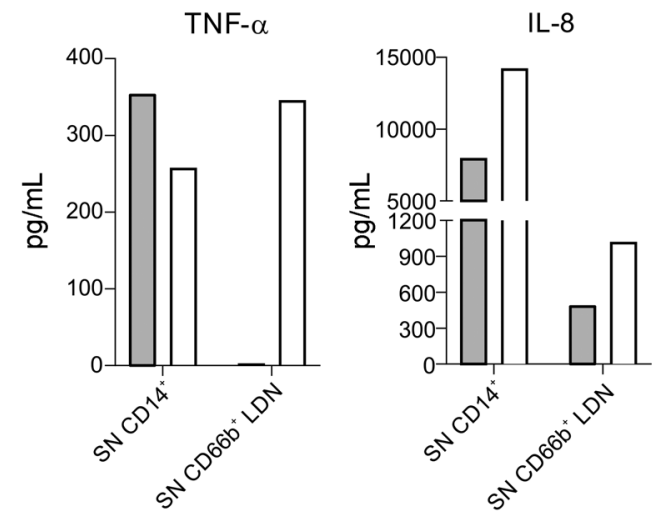

Figure 3. Baricitinib treatment alters the immune-suppressive abilities and distribution of myeloid cells during the recovery phase. (A) Plasma from a patient with COVID-19 enrolled in the baricitinib treatment arm who was admitted to the ICU during treatment was analyzed at $t 0$ (baseline), $t 4$ (4 days after treatment), and $t 7$ (7 days after treatment) to assess proinflammatory cytokine levels by automated immunoassay system. Peripheral blood from the same patient was tested while the patient was in the ICU and after leaving the ICU (No ICU). (B) Monocytes (CD14+), LDNs (CD66b+), and NDNs $\left(\mathrm{CD}_{6} \mathrm{~b}^{+}\right)$were isolated from the peripheral blood. The immune-suppressive abilities of either enriched myeloid cells or their conditioned media were tested in functional assays to assess T cell activation and are shown as the percentage of suppression. Monocyte (C) and neutrophil (D) subsets were evaluated by flow cytometry and are reported as the number of cells per microliter. (E) Cytokines released in the conditioned media by CD14+ and CD66b ${ }^{+}$LDNs were quantified by multiplex ELISA. (B-D) Data are reported as the mean \pm SEM. $P$ values in B-D were determined by 2-tailed Student's $t$ test. SN, supernatant.

as IL-8 levels had a tendency to increase during the same time frame (Figure 3A). We then evaluated the capacity of the isolated myeloid cells to suppress the proliferation of activated $\mathrm{T}$ cells. As shown in Figure 3B, the suppressive activity of monocytes (CD14 ${ }^{+}$ cells), as well as of their supernatants, decreased when the patient left the ICU, while it was maintained in $\mathrm{CD} 66 \mathrm{~b}^{+} \mathrm{LDNs}$ on a percell basis. As expected, the $\mathrm{CD} 6 \mathrm{~b}^{+} \mathrm{NDN}$ fraction was poorly suppressive. Although the total count of monocytes seemed to be unaffected by the treatment, we observed an opposite trend in the distribution among monocyte subsets, defined as classical (CD14 ${ }^{\text {hi }}$ $\left.\mathrm{CD} 16^{10 / \mathrm{dim}}\right)$ and nonclassical (CD14 $\left.{ }^{\mathrm{lo} / \mathrm{dim}} \mathrm{CD} 16^{\mathrm{hi}}\right)$ cells, with the former decreasing and the latter increasing during the time points analyzed (Figure 3C). Similarly, baricitinib treatment did not alter the total neutrophil count, but rather shifted the distribution from the LDN fraction, which decreased during the treatment, to the NDN fraction, which, conversely, increased (Figure 3D). Of note, when we assessed the cytokine content in the conditioned media obtained from the immune-suppressive cell populations, i.e., the monocytes and LDNs analyzed in Figure 3B, we noticed that, overall, monocytes secreted a greater amount of cytokines than did LDNs and that, on a per-cell basis, the breadth of cytokine release was generally higher in monocytes (Figure 3E), consistent with published data about the contribution of monocytes to the cytokine storm $(12,13)$.

\section{Discussion}

We report here the effect of short-term treatment with baricitinib on regularizing the immune landscape in patients with COVID-19. Considering that this treatment can be orally administered to patients outside the hospital setting, the impact on limiting the negative consequences of SARS-CoV-2 during the pandemic spread might be of utmost relevance for the global health care sys- 
tem. The clinical benefits for patients treated with baricitinib and the potential mortality risk were evaluated in a recent preliminary retrospective, multicenter study (14) and will have to be further established in the ongoing randomized clinical trials. Our findings also indicate that there might be a substantial advantage in targeting the STAT3 pathway. Many viruses might have developed strategies to trigger STAT3 signaling to dampen the antiviral innate immune response during the acute phase, either by preventing IFN responses or triggering the negative immune-regulatory effects of IL-6 and IL-10 $(11,15)$. Indeed, the STAT3 pathway is relevant for the production of some cytokines during the CRS, including but not limited to IL-6 and IL-10 (16). It is important to highlight how baricitinib also affects IL- $1 \beta$ and TNF- $\alpha$ levels, which are traditionally considered to be under transcriptional regulation by molecular pathways distinct from JAK1/JAK2, i.e., inflammasome and NFAT/NF- $\kappa$ B pathways, respectively (17-19). From the immune standpoint, a reduction of STAT3 in NK cells promotes a consistent increase in perforin and granzyme B, improving NK-mediated surveillance against pathogens (20). On the other hand, preventing STAT3 phosphorylation in monocytes and neutrophils affects the ability to produce and release proinflammatory cytokines (16) as well as their immunosuppressive properties on $\mathrm{T}$ lymphocytes (21). Moreover, STAT3 in cytotoxic CD8 ${ }^{+} \mathrm{T}$ cells controls lymphocyte differentiation from an effector to a long-term central memory phenotype (22).

In the context of the health crisis in northern Italy, where the hospital capacity was quickly overwhelmed by a heavy load of patients with severe COVID-19, the decision to use baricitinib was based on several practical considerations. The first consideration took into account not only the pathogenesis of the disease but also the current availability of the drug for RA therapy. Although its use for COVID-19 treatment was off-label, baricitinib treatment in humans was already authorized by the Italian regulatory authorities as a therapeutic for RA, thereby facilitating its immediate use. A second consideration was related to the pharmacokinetics and pharmacodynamics of the drug. Since early intervention on the cytokine cascade might prevent the progression to virus-induced damage, we administered a loading dose to gauge the drug's effect. Phase I pivotal studies of healthy volunteers taking standard daily doses of $4 \mathrm{mg}$ baricitinib demonstrated that a plasma steady-state concentration of the drug is normally achieved within 48 hours (23). To achieve this concentration in the first 24 hours, a double dose ( 4 mg every 12 hours for 48 hours) was planned, followed by the standard dose on the subsequent days. Upon reaching the steady-state concentration, the inhibition of STAT3 phosphorylation occurs within 2 to 4 hours (23). In this way, the action of the drug on the STAT3 target should already be guaranteed within the first 24 hours. The present data confirm that an early effect was indeed reached in T lymphocytes, NK cells, monocytes, and neutrophils (Supplemental Figure 1). A third consideration involved the safety profile of baricitinib $(7,8)$. This aspect was of crucial importance in the context of an off-label use of the drug.

This study has several limitations. First, there are missing data for some outcomes (i.e., immunological parameters for some of the enrolled patients). Second, the follow-up time was relatively short because of the disease timeline. Third, this study was not double-blinded. Fourth, the ability of baricitinib to modify the immune-suppressive features of myeloid cells was shown in only 1 patient from whom a sufficient number of biological samples were collected over the course of the disease.

In conclusion, our data suggest the potential effectiveness of short-term treatment with baricitinib for patients with ongoing SARS-CoV-2 infections in the absence of effective antiviral treatments or vaccines.

\section{Methods}

Complete details on methods are provided in the Supplemental Methods.

Patients. From March 25 to April 18, 2020, patients admitted to the Unit of Internal Medicine at the University Hospital of Verona and Pederzoli Hospital of Peschiera with a diagnosis of COVID-19 pneumonia, confirmed by a nasal swab positive for SARS-CoV-2 determined by RT-PCR assay, were consecutively enrolled in the study. A group of healthy donors $(n=6)$ were also enrolled as controls.

A group of study participants $(n=20)$ were treated with baricitinib according to an off-label use of the drug. These patients were not considered for the randomized, multicenter clinical trial that will start recruitment. The use of the drug has been proposed on the basis of a therapeutic protocol with stringent inclusion criteria. In particular, a clinical onset of symptoms not exceeding 9 days and the presence of interstitial lung involvement not exceeding $50 \%$ on chest x-ray or $\mathrm{CT}$ were required for patients to receive baricitinib therapy. Exclusion criteria included the presence of active malignancies and/or immunodeficiency, cardiovascular disease with recent myocardial infarction or stroke, as well as thrombophilia or deep venous thrombosis or pulmonary thromboembolism. Additional exclusion criteria included the presence of chronic kidney disease with renal failure, cirrhosis with a Child-Pugh score of C, or the presence of anemia or severe neutropenia or lymphocytopenia (Supplemental Table 1).

Clinical features during treatment were recorded for all patients included in the study. Flow cytometric, cytokine, and serology assays were performed in a subgroup of patients on the basis of biological sample availability.

Study assessment. This off-label treatment was evaluated using laboratory values including serum CRP concentrations and the oxygenation index $(\mathrm{P} / \mathrm{F})$, as well as immunological parameters including serum cytokine levels (IL-6, IL-1 $\beta$, TNF- $\alpha$ and IL-8), serology, and absolute numbers of different immune cell populations from the day of enrollment out to day 7. Further details regarding flow cytometry, cell purification assays, ELISAs, and cellular assays are provided in the Supplemental Methods. We quantified the incidence of key clinical elements such as the need for oxygen flow, the $\mathrm{P} / \mathrm{F}$ ratio, the radiology score calculated on the basis of the percentage of interstitial lung involvement $(0=$ no interstitial lung involvement; 1 = interstitial involvement $<20 \%$; 2 = parenchymal involvement between $20 \%$ and $50 \% ; 3=$ parenchymal involvement between $50 \%$ and $70 \% ; 4=$ interstitial involvement $>75 \%$ ); the respiratory rate as well as ARDS incidence; the duration of hospitalization, and death.

Statistics. The clinical analysis at baseline $(t 0)$ included all patients enrolled in the study for whom laboratory tests were available (see Tables 1 and 2 and Supplemental Table 1). Given the nature of this off-label program, there are some missing data. For treatment period analysis, we considered only those patients who were still alive and for whom the clinical and laboratory data for at least 2 time points were available. Analysis of the duration of hospitalization was performed 
for all patients except for those who died. Quantitative variables are expressed as the median and IQR, qualitative ones as percentages. For pairwise comparisons, significance differences were determined by the Mann-Whitney $U$ test for quantitative variables and the Fisher exact test for categorical variables. The Wilcoxon signed rank test was used to test the equality of observation. This test was applied to evaluate differences at $t 0$ versus $t 4$ and at $t 0$ versus $t 7$ for the respiratory rate, $\mathrm{P} / \mathrm{F}, \mathrm{FiO}_{2}, \mathrm{CRP}$ levels, and fever for each patient. Statistical significance was set at a $P$ value of less than 0.05 , and clinical analyses were performed using STATA, version 16.0 (StataCorp). For statistical laboratory analyses, paired comparisons by 2-tailed Student's $t$ test or 1-way ANOVA for repeated measures were performed using GraphPad Prism, version 8.4.2 (GraphPad Software). A P value of less than 0.05 was considered significant.

Study approval. The study was designed as an observational longitudinal trial and approved by the Ethics Committee for Clinical Experimentation, Department of Hospital Medical Management, Hospital Trust of Verona in Verona, Italy (protocol 17963; principal investigator, Vincenzo Bronte; ClinicalTrials.gov identifier NCT04438629). All clinical investigations were conducted according to Declaration of Helsinki principles, and informed consent was obtained from all study participants.

\section{Author contributions}

V. Bronte, CL, and OO designed the research. V. Bronte and SU coordinated the study. ET, V. Batani, EP, KD, SF, FP, DR, PB, MR, AC, WM, CL, and OO collected clinical specimens and data. LT performed the statistical analysis. AV, FDS, RT, AF, LP, CM, and S.
Canè completed flow cytofluorimetric data acquisition and analysis. VP, FH, and MI analyzed serum and plasma cytokine levels. S. Canè and RMB conducted functional analyses. FF and PGP performed serological analysis and prepared key reagents. SU, ET, FDS, RT, and S. Caligola analyzed the results. SU, ET, S. Canè, CL, $\mathrm{OO}$ and $\mathrm{V}$. Bronte wrote the manuscript.

\section{Acknowledgments}

We dedicate this work to the memory of the health care workers who have given their lives in the care of patients with COVID-19. We thank all the patients who participated in this study and their families. We also thank all the personnel involved in the patients' care and assistance. We wish to thank the following members of the Immunology Section of Verona University Hospital, who worked during the pandemic: Morena Martini, Fiorenza Paiola, Elena Lucchini, Claudia Pizzoli, Elena Chiesa, Oretta Gabrielli, Nadia Brutti, Monica Brentegani, Elisabetta Gallo, Giulio Fracasso, Tiziana Cestari, Ornella Poffe, and Cristina Anselmi for their excellent technical work; Cristina Frusteri, Giovanna Zanoni, Silvia Sartoris, Riccardo Ortolani, and Selena Gomirato for their help with the management of immunological data; Daniel Lovato, Antonella Valentini, and Claudia Italia for their administrative support.

Address correspondence to: Vincenzo Bronte, Immunology Section, Department of Medicine, University and Hospital Trust of Verona, P.le Scuro 10, 37134 Verona, Italy. Phone: 390458124007; Email: vincenzo.bronte@univr.it.
1. World Health Organization. Coronavirus disease (COVID-19) outbreak. https://www.who.int. Accessed June 26, 2020.

2. Zhang B, et al. Clinical characteristics of 82 death cases with COVID-19. PLos One. 2020;15(7):e0235458.

3. Moore JB, June CH. Cytokine release syndrome in severe COVID-19. Science. 2020;368(6490):473-474.

4. Mehta P, et al. COVID-19: consider cytokine storm syndromes and immunosuppression. Lancet. 2020;395(10229):1033-1034.

5. Tay MZ, Poh CM, Rénia L, MacAry PA, Ng LFP. The trinity of COVID-19: immunity, inflammation and intervention. Nat Rev Immunol. 2020;20(6):363-374.

6. Genovese MC, et al. Baricitinib in patients with refractory rheumatoid arthritis. $\mathrm{N} \mathrm{Engl} \mathrm{J} \mathrm{Med.}$ 2016;374(13):1243-1252.

7. Honda S, Harigai M. The safety of baricitinib in patients with rheumatoid arthritis. Expert Opin Drug Saf. 2020;19(5):545-551.

8. Bechman K, et al. A systematic review and meta-analysis of infection risk with small molecule JAK inhibitors in rheumatoid arthritis. Rheu- matology (Oxford). 2019;58(10):1755-1766

9. Richardson P, et al. Baricitinib as potential treatment for 2019-nCoV acute respiratory disease. Lancet. 2020;395(10223):e30-e31.

10. Wen W, et al. Immune cell profiling of COVID19 patients in the recovery stage by single-cell sequencing. Cell Discov. 2020;6(1):31.

11. Thevarajan I, et al. Breadth of concomitant immune responses prior to patient recovery: a case report of non-severe COVID-19. Nat Med. 2020;26(4):453-455.

12. Jeong J, Suh Y, Jung K. Context drives diversification of monocytes and neutrophils in orchestrating the tumor microenvironment. Front Immunol. 2019;10:1817.

13. Merad M, Martin JC. Pathological inflammation in patients with COVID-19: a key role for 439 monocytes and macrophages. Nat Rev Immunol. 2020;20(6):355-362.

14. Cantini F, Niccoli L, Matarrese D, Nicastri E, Stobbione P, Goletti D. COVID-19: a pilot study on safety clinical impact. JInfect. 2020;81(2):318-356.

15. Kuchipudi SV. The complex role of STAT3 in viral infections. J Immunol Res. 2015;2015:272359.

16. Yu H, Pardoll D, Jove R. STATs in cancer inflam- mation and immunity: a leading role for STAT3. Nat Rev Cancer. 2009;9(11):798-809.

17. Schroder K, Tschopp J. The inflammasomes. Cell. 2010;140(6):821-832.

18. Taniguchi K, Karin M. NF-kB, inflammation, immunity and cancer: coming of age. Nat Rev Immunol. 2018;18(5):309-324.

19. Falvo JV, Tsytsykova AV, Goldfeld AE. Transcriptional control of the TNF gene. Curr Dir Autoimmun. 2010;11:27-60.

20. Gotthardt D, et al. Loss of STAT3 in murine NK cells enhances NK cell-dependent tumor surveillance. Blood. 2014;124(15):2370-2379.

21. Trovato R, et al. Immunosuppression by monocytic myeloid-derived suppressor cells in patients with pancreatic ductal carcinoma is orchestrated by STAT3. J Immunother Cancer. 2019;7(1):255

22. Siegel AM, et al. A critical role for STAT3 transcription factor signaling in the development and maintenance of human $\mathrm{T}$ cell memory. Immunity. 2011;35(5):806-818.

23. Shi JG, et al. The pharmacokinetics, pharmacodynamics, and safety of baricitinib, an oral JAK $1 / 2$ inhibitor, in healthy volunteers. J Clin Pharmacol. 2014;54(12):1354-1361. 